\title{
THE ENERGY RECOVERY LINAC (ERL) AS A DRIVER FOR X-RAY PRODUCING INSERTION DEVICES*\#
}

\author{
I. V. Bazarov, D. H. Bilderback, S. M. Gruner, H. S. Padamsee, R. Talman, M. Tigner, \\ Cornell University, Ithaca, NY 14853, USA \\ G. A. Krafft, L. Merminga, C. K. Sinclair, TJNAF, Newport News, VA 23606, USA
}

\begin{abstract}
Storage rings have served well as $\mathrm{x}$-ray sources, achieving continued increases in flux and brilliance. While further improvements in performance may still be expected, the performance of these machines is constrained by radiation fluctuations and by the Touschek effect. The practical effect of the radiation fluctuations is to limit the minimum 6D emittance of the rings in general, and particularly the bunch length. The Touschek effect limits the lifetime and the bunch charge density. These effects can be ameliorated by using a linac to accelerate the beam to the requisite energy before passing it through undulators to produce the $\mathrm{x}$-rays. If the beam is discarded after producing $\mathrm{x}$-rays, radiation and collision effects are no longer limiting. This approach has not been used because prohibitive amounts of energy would be required to produce the beam. However, by using energy recovery in a superconducting linac, the energy can be recycled to accelerate new electrons. This would allow the use of currents comparable to those in storage rings, but with superior emittance, bunch length and flexibility. The concept of a $5-7 \mathrm{GeV}$ facility using this principle is described and parameter goals given.
\end{abstract}

\section{INTRODUCTION}

Synchrotron Radiation (SR) x-ray sources have come a long way from the first parasitic uses of high energy physics machines to specially dedicated $3^{\text {rd }}$ generation SR facilities of the present day [1]. Improvements in electron storage rings and insertion devices (IDs) have led to an exponential increase in brilliance for each successive generation of the x-ray sources. As of today, however, the technology of storage rings has reached a mature stage, resulting in the fact that $3^{\text {rd }}$ generation SR facilities are capable of providing the beam quality close to the limit determined by the equilibrium between quantum excitation and radiation damping in these machines. This equilibrium sets limits on transverse emittance and bunch length while the space charge related Toushcek effect tends to limit beam lifetime. Consequently, it is increasingly difficult to meet the fast growing demands of the X-ray scientific community with SR sources based on storage ring technology.

On the other hand, it has been long known that linear accelerators can produce both low emittance (with normalized emittance determined at the injection point)

\footnotetext{
*Supported by NSF Coop. Agrmts NSF PHY 9809799, DMR-9713424

"Supported by US DOE No. DE-AC05-84ER40150
}

and very short bunches because the limitations set by equilibrium are no longer an issue. As a result, several proposed large projects are based on linear accelerators (e.g. [2, 3]). Because of the enormous beam power required for high current operation, linear accelerators are forced to be relatively low average current machines (on the order of milliAmpere or less). While this is not a constraint for many applications, for continuous SR sources it is desirable to have a high quality, high current beam. A mechanism for recycling beam energy via the use of superconducting linacs was proposed long ago by Tigner [4] for colliding beam machines. This concept of energy recovery at modest average current was first verified at the Stanford SCA/FEL [5]. More recently, an IR FEL Demo facility at TJNAF has demonstrated energy recovered beam recirculation at $5 \mathrm{~mA}$, the highest average current to date [6]. The considerable improvement over the last decade in the beam quality available from laserdriven photoinjectors, and in superconducting $\mathrm{rf}$ (srf) cavities capable of high accelerating gradients now make the use of energy recovery for a continuous SR source (e.g., an ERL) very attractive. Hence, we have proposed a high average current $(100 \mathrm{~mA})$ multi-GeV ERL as a driver for an X-ray SR facility [7].

\section{GENERAL DESCRIPTION OF ERL}

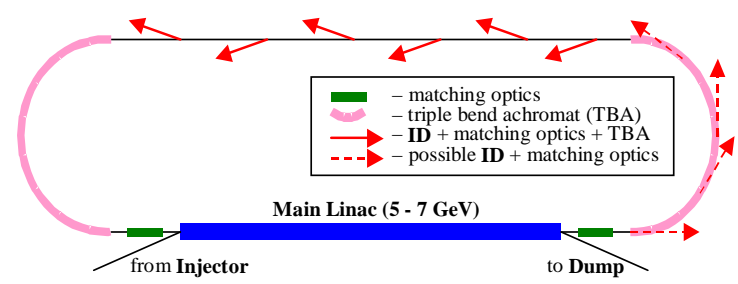

Fig. 1: Conceptual layout of ERL.

A conceptual layout for an ERL is shown in Fig. 1. The essential parts of the facility are the photoinjector, the main accelerator, the SR transport loop and undulators for x-ray production. A $10 \mathrm{MeV}$ electron beam with small normalized transverse emittance is introduced into the main srf linac from the injector. The srf structure accelerates the electrons to 5-7 GeV. Subsequently, the beam is taken through the recirculation transport loop where it is used to produce x-rays. The beam is then returned to the linac with a $180^{\circ} \mathrm{rf}$ phase offset for energy recovery. In the linac the recirculated electrons give back their energy which is then being used for the acceleration of successive bunches. Finally, the low energy beam exits 
the main linac and is diverted to a beam dump with a lowfield dipole magnet.

Table 1: ERL parameter list.

\begin{tabular}{lcc}
\hline Parameter & Value & Unit \\
\hline Beam Energy & $5-7$ & $\mathrm{GeV}$ \\
Average Current & $100 / 10$ & $\mathrm{~mA}$ \\
Fundamental frequency & 1.3 & $\mathrm{GHz}$ \\
Charge per bunch & $77 / 8$ & $\mathrm{pC}$ \\
Injection Energy & 10 & $\mathrm{MeV}$ \\
Normalized rms emittance & $\leq 2 / 0.2$ & $\mathrm{~mm}-\mathrm{mrad}$ \\
Energy spread (rms) & $0.02-0.3$ & $\%$ \\
Bunch length in IDs (rms) & $0.1-2$ & $\mathrm{ps}$ \\
Total radiated power (typical) & 400 & $\mathrm{~kW}$ \\
X-ray brilliance & $10^{22}$ & $* *$ \\
\hline ** Photon / (sec $\left.\cdot \mathrm{mrad}^{2} \cdot \mathrm{mm}^{2} \cdot 0.1 \% \mathrm{BW}\right)$ &
\end{tabular}

The injector will be based on a DC gun with a laserdriven negative affinity photocathode. Srf cavities will be used to boost the energy of the beam to $10 \mathrm{MeV}$ where the space charge effects are significantly reduced. Each rf bucket of the $1.3 \mathrm{GHz}$ srf cavities will be filled, corresponding to $77 \mathrm{pC}$ charge per bunch for $100 \mathrm{~mA}$ beam current. The relatively low charge per bunch is chosen to alleviate problems intrinsic to the higher charges, in particular, coherent synchrotron radiation (CSR), space charge, and wakefields effects on the $6 \mathrm{D}$ emittance. In the injector the bunches undergo preliminary compression to about $0.7 \mathrm{~mm}$ rms bunch length before they are deflected into the main linac. Simulations indicate that normalized emittances as good as $1 \mathrm{~mm}$-mrad (rms) can be expected from the injector. The capacity to operate the injector with lower charge per bunch but improved transverse emittances is also envisioned.

The main linac consists of 9-cell TESLA type srf cavities with an accelerating gradient of $20 \mathrm{MV} / \mathrm{m}$. Quadrupole focusing in the main linac suppresses the multipass beam break-up (BBU) instability and allows small betatron functions for both the accelerated and energy recovered beams [8]. In the simplest configuration (Fig. 1) a single linac is used. Alternatives, such as multiloop acceleration, in which the beam is accelerated through the linac several times, have been suggested. However, the challenges of such multi-loop designs appear to be significant. In particular, with the higher order mode (HOM) damping presently achieved [3], the simulated BBU threshold current is much lower than that of a single-loop design.

The SR transport loop hosts the undulators and beam lines required to produce SR. The transport loop is designed in such a way as to minimize various causes of emittance dilution (e.g., quantum excitation, CSR effects). The ability to perform longitudinal phase space manipulations in the transport loop is also a feature.

Optimal use of an ERL will require undulators with a very large number of periods and will tax the capabilities of present day x-ray optics. Although one can imagine many types of specialty undulators, two IDs that seem to be suitable for much of the suggested $\mathrm{x}$-ray science [9] of the ERL are an undulator of 25 meter length (segmented, $1.7-\mathrm{cm}$-period) and a 2 meter undulator $(1.7$ to $3.3 \mathrm{~cm}$ period length). When combined with the low emittance of the ERL, $25 \mathrm{~m}$ long ID would provide unprecedented brilliance and flux.

A sample parameter list for an ERL $\mathrm{x}$-ray source is presented in Table 1. More details on the characteristics of x-rays from this ERL (brilliance curves, comparison with some of the existing and proposed SR facilities, coherence properties, etc.) can be found in [10].

\section{ACCELERATOR PHYSICS \& TECHNOLOGY CHALLENGES}

Considerable investigation of the accelerator physics issues involved will be required and technological advancements attained in order to achieve the flux, brilliance, and time structures that appear to be possible with the ERL. The accelerator physics issues revolve largely around emittance production and preservation and have implications for all parts of the accelerator, from the laser to the gun itself, the booster section, the main linac, the transport lines and the undulators. Technological issues center on cathode longevity, high gradient CW operation of superconducting cavities well beyond current standards, and the very high efficiency energy recovery demanded for economical and stable operation. In addition, diagnostics and beam control for such a bright and high power beam will be significant challenges.

\subsection{Accelerator Physics Challenges}

To achieve the goal of $\leq 2 \mathrm{~mm}$-mrad normalized emittance in the undulators, it will be necessary to achieve $\leq 1.5 \mathrm{~mm}$-mrad out of the injector and less than that from the gun. Thus, very careful emittance compensation [11] must be realized at the low energy end where the beam is most vulnerable and effects of non-inertial space charge and CSR emittance dilution [12] must be strictly minimized. These are matters under active research in the accelerator community and no completely reliable computer codes for calculating these effects are as yet available. Wakefields in the cavities and beam lines will also present a challenge to emittance preservation and are, to a lesser extent, still research topics in the field.

Another topic of great importance, related to the above, is halo formation on the beam and its control or removal. With such a high power beam, halo and its loss to the walls will be inimical to stable operation and high efficiency energy recovery. Prediction and measurement of halos at various positions in the loop will be an important datum in the design of a full scale facility.

\subsection{Accelerator Technology Challenges}

The photoinjector source is at the heart of the facility since it determines the maximum achievable flux and brilliance. Various source technologies are being studied for their potential suitability for an ERL aimed ultimately 
at x-ray production. Initial surveys and calculations convince us that the DC, laser-driven photoemission gun is the most likely to be successful. Selecting the optimum cathode material and assuring adequate operating life under high current operating conditions present significant challenges. Minimization of dark current and operation at the highest practical cathode voltages will require developments in insulators, cathode materials, and the maintenance of extreme vacuum conditions.

The superconducting booster section of the injector will also require much development. The need to minimize emittance-diluting asymmetries while coupling $1 \mathrm{MW}$ to the beam in a flexible way so that $\mathrm{rf}$ focusing and $\mathrm{rf}$ bunching can be accomplished without destroying emittance compensation is more demanding than in any existing system.

The main linac posited for the ERL operates at levels well beyond existing technology. The economic optimum indicates that approximately $20 \mathrm{MV} / \mathrm{m}$ is a desirable operating gradient. This gradient has been routinely achieved in pulsed operation and with relatively small average beam currents. It must be demonstrated that the necessary gradients and $\mathrm{Q}$ values can be maintained under $\mathrm{CW}$, high average current operating conditions. Furthermore, to avoid emittance dilution from BBU driven by HOMs in the cavities, one will have to achieve heavier HOM damping than is now the case in srf structures without compromising the achievable Q and gradient. $\mathrm{CW}$ operation at high average current results in high HOM power, which must be extracted from the cryogenic environment with high efficiency. This demands not only innovations in the off-beam-line HOM couplers but also development of on-line absorbers to catch the HOM power that propagates down the beam line. Careful measurements will be needed to assure that these criteria can be met and maintained under operating conditions. Economic operation of the main linac will benefit from using high external Q values, thereby minimizing the klystron power required. The challenge here is the microphonic detuning inevitably present in the system. Development of an active feedback system that can operate over the full dynamic range of beam current and cavity gradient must therefore be a priority in the development of ERL facility.

An important feature of the ERL is the possibility of providing sub-picosecond bunches to the undulators. Avoiding the severe wakefield consequences of such short bunches in the injector and linac requires that the short bunches be obtained at the highest energy by magnetic compression. The accelerator physics and technology of effecting this without degradation of the beam properties is an important subject which requires further exploration before one can design the needed system for a full scale facility with confidence.

Measuring the beam properties with the accuracy needed in the face of the enormous average beam power will require non-intercepting methods that are robust and easily read out for tuning and feedback control. Optical methods analyzing incoherent and coherent synchrotron radiation at various locations around the loop are promising [13] but will need demonstration in this very low normalized emittance context. Likewise, measuring halo in the presence of such high average power will be a challenging task. Reliable methods for doing so must be developed as tuning and diagnostic tools.

\section{OUTLOOK}

Given the large number of parameters to be pushed beyond current levels, it is proposed to build a prototype in which many of these parameters can be demonstrated and most of the accelerator technology issues outlined in the previous section will be addressed. The prototype will have a full power injector, a linac and a return transport loop along with the beam manipulation and measurement equipment necessary for exploration of all anticipated operating modes of an eventual full size facility. The photoinjector is essentially that required for a full scale machine to be able to demonstrate the needed current (i.e. $100 \mathrm{~mA}$ ), cathode lifetime and emittance capabilities ( $\leq 2$ $\mathrm{mm}$-mrad). The linac is likewise intended to be a short section of the srf structure needed for a full facility, allowing evaluation of all the relevant technologies. The main linac, capable of producing $100 \mathrm{MeV}$, will consist of five $1 \mathrm{~m}$ srf cavities. A $100 \mathrm{MeV}$ linac and a $5 \mathrm{MeV}$ injector provide an energy ratio of 20 , which is deemed large enough to explore instability thresholds and investigate beam physics issues in the linac. Effects peculiar to emittance preservation of sub-picosecond bunches (CSR, wakes, non-inertial space charge) will be studied experimentally and various codes predicting these phenomena will be benchmarked. Detailed exploration of the prominent accelerator physics issues with the prototype will be an indispensable step supporting the construction of a future ERL x-ray source.

\section{REFERENCES}

[1] H. Winick, IEEE Proc. 1989 Part. Accel. Conf., p. 711 (1989)

[2] R. Tatchyn et al., NIM A 375, p. 274-83 (1996)

[3] TESLA TDR, DESY 2001-011, March 2001

[4] M. Tigner, Nuovo Cimento 37, p. 1228-31 (1965)

[5] T. I. Smith et al., NIM A 259, p. 1-7 (1987)

[6] G. R. Neil et al., NIM A 429, p. 27-32 (1999)

[7] S. M. Gruner et al., White Paper, http://erl.chess.cornell.edu/Papers/Papers.htm

[8] I. V. Bazarov et al., these proceedings

[9] D. Bilderback et al., SR News 14, p. 12-21 (2001)

[10] Q. Shen, CHESS TM 01-002,

http://erl.chess.cornell.edu/Papers/Papers.htm

[11] B. E. Carlsten, NIM A 285, p. 313-319 (1989)

[12] R. Li, IEEE Proc. 1999 Part. Accel. Conf. 1, p. 118-

122 (1999)

[13] R. Lai, Phys. Rev. E 50, p. R4294-R4297 (1994) 